\title{
BMJ Open Perceived working mechanisms of psychosomatic therapy in patients with persistent somatic symptoms in primary care: a qualitative study
}

Margreet S H Wortman (10, , ${ }^{1,2}$ Tim C Olde Hartman, ${ }^{3}$ Johannes C van der Wouden, ${ }^{1}$ Sarah Dankers, ${ }^{1}$ Bart Visser, ${ }^{2}$ Willem J J Assendelft, ${ }^{3}$ Henriëtte E van der Horst (D) ${ }^{1}$

To cite: Wortman MSH, Olde Hartman TC, van der Wouden JC, et al. Perceived working mechanisms of psychosomatic therapy in patients with persistent somatic symptoms in primary care: a qualitative study. BMJ Open 2022;12:e057145. doi:10.1136/ bmjopen-2021-057145

- Prepublication history and additional supplemental material for this paper are available online. To view these files, please visit the journal online (http://dx.doi.org/10.1136/ bmjopen-2021-057145).

Received 06 September 2021 Accepted 23 December 2021

Check for updates

(C) Author(s) (or their employer(s)) 2022. Re-use permitted under CC BY-NC. No commercial re-use. See rights and permissions. Published by BMJ.

${ }^{1}$ Department of General Practice, Amsterdam UMC Locatie VUmc, Amsterdam, The Netherlands ${ }^{2}$ Faculty of Health, Centre of Expertise Urban Vitality, Amsterdam University of Applied Sciences, Amsterdam, The Netherlands

${ }^{3}$ Department of Primary and Community Care, Radboud University Nijmegen, Nijmegen, The Netherlands

Correspondence to Margreet S H Wortman; m.wortman@amsterdamumc.nl

\section{ABSTRACT}

Objectives To explore the perceived working mechanisms of psychosomatic therapy according to patients with persistent somatic symptoms (PSS) and their psychosomatic therapists.

Design Qualitative study using semistructured face-toface interviews and focus groups. All interviews were audiorecorded, transcribed verbatim and analysed, by two researchers independently, based on the thematic analysis. Setting Alongside a randomised controlled trial to establish the (cost-)effectiveness of psychosomatic therapy in patients with PSS in primary care, we conducted a process evaluation with a qualitative study. Patients were recruited in general practice in three regions in the Netherlands.

Participants Interviews were conducted with twenty patients with PSS who received psychosomatic therapy and 25 psychosomatic therapists. In addition, two focus groups were conducted with six and seven psychosomatic therapists, respectively.

Intervention Psychosomatic therapy, delivered by specialised exercise and physical therapists, is a multimodal and tailored treatment based on the biopsychosocial model.

Outcome measures Experiences, opinions and views from patients' and therapists' perspective on psychosomatic therapy were identified.

Results A total of 37 interviews with patients, 25 interviews and two focus groups with therapists were analysed. Three main themes emerged from the data of the patients: (1) continuous alternation of psychosocial conversations and body-oriented exercises; (2) awareness of body-mind connection and (3) good relationship with therapist. Four main themes emerged from the data of the therapists (1) building rapport; (2) continuously searching for common ground; (3) making patients aware of the interaction between body and mind; and (4) continuous alternation between exploration and treatment.

Conclusion According to patients as well as therapists, the continuous alternation of psychosocial conversations and body-oriented exercises to provide awareness of the interaction between body and mind are the perceived working mechanism of psychosomatic therapy. Therapeutic alliance and finding common ground between patient and therapist are prerequisites for the success of psychosomatic therapy.
Strengths and limitations of this study

- This qualitative study highlights the opinions and views from therapists' and patients' perspective on the elements contributing to the effects of psychosomatic therapy in patients with persistent somatic symptoms (PSS).

- Patients were recruited in general practice and we used a purposive sampling strategy to ensure variation in gender, age, region, symptom intensity, number of treatment sessions and therapist and in that regard represent a broad sample of patients with PSS.

- We used triangulation of sources to collect and analyse the data, following an iterative process and modification of procedures in response to evolving study findings.

- A majority of the patients started the psychosomatic therapy without a specific request for help and without expectations, this may have influenced their experiences with the psychosomatic therapy.

Trial registration number NL7157 (NTR7356).

\section{INTRODUCTION}

Persistent somatic symptoms (PSS), such as headache, dizziness, fibromyalgia and chronic fatigue syndrome, are symptoms that persist longer than usual and, either by their character or following the negative results of clinical examination, cannot be attributed to a disease. PSS are highly prevalent in primary care. ${ }^{1}$ Patients often experience functional impairment, interference with functioning at work $^{2}$ and a reduced quality of life. ${ }^{34}$

A wide variety of interventions are used for the treatment of PSS such as pharmacological treatments (eg, antidepressants), cognitivebehavioural therapy (CBT), psychodynamic interpersonal psychotherapy, ${ }^{5}$ relaxation therapies and physical therapies. However, these interventions showed only small to moderate 
effects in reducing somatic symptoms. ${ }^{6-9}$ Moreover, most patients with PSS do not easily accept psychological treatment ${ }^{710}$ because they have strong views about the biological nature of their symptoms and view the suggestion of a more psychological approach as invalidating. ${ }^{11}$

Research suggests that multimodal treatments, which address both physical and cognitive behavioural aspects of PSS at the same time, may be more acceptable for and effective in patients with PSS. ${ }^{72}$ However, there has been little research that primarily focuses on the efficacy of a multimodal treatment for patients with PSS in primary care. ${ }^{71314}$ In addition, studies did not explore the (causal) mechanisms underlying the interventions. ${ }^{11}$

In the Netherlands, there are specialised exercise and physical therapists with a special interest in PSS who deliver psychosomatic therapy. ${ }^{15} 16$ Psychosomatic therapy is a multimodal, stepped-care and tailored treatment based on the biopsychosocial model in which illness is viewed as a result of interacting mechanisms at the biological, psychological, behavioural, interpersonal and environmental levels. This implies that patients' symptoms, illness beliefs, concerns, illness behaviour and social environment are addressed. It includes the following elements: psycho-education, body-focused therapy with relaxation therapy and mindfulness, cognitive-behavioural approaches and activating therapy. The overall aim of the treatment is to improve patients' functioning. According to the Dutch College of general practitioners' (GP) guideline for treating PSS ${ }^{17}$ psychosomatic therapy is regarded as an important treatment option for patients with moderate PSS. It has the potential to result in symptom improvement and seems to be acceptable for patients with PSS. ${ }^{18}$

Currently, we are performing a large randomised controlled trial (RCT), the CORPUS study, to establish the (cost-)effectiveness of psychosomatic therapy in patients with PSS in primary care. ${ }^{19}$ Because this therapy is a complex, multimodal intervention, we conducted a process evaluation alongside the RCT. The aim of this study is to explore patients' and therapists' experiences with and views and opinions on the working mechanisms of the psychosomatic therapy.

\section{METHODS \\ Design}

In the CORPUS study, patients were recruited in general practice in three regions in the Netherlands. Eligible patients (aged 18-80 years) with one or more 'Robbins List' symptoms ${ }^{20}$ (see online supplemental appendix 1) who visited their GP frequently over the past 2 years were identified by a search strategy in the electronic medical records. Potentially eligible patients received information about the study and the Patient Health Questionnaire-15 (PHQ-15) by mail from their GP. ${ }^{20-22}$ Patients with a PHQ-15 score of $\geq 5$, which means that they have at least medium somatic symptom severity, ${ }^{21}{ }^{22}$ received an informed consent form. Patients who agreed to participate were individually randomised to the intervention group (psychosomatic therapy as an adjunct to usual care) or to usual care (see online supplemental appendix 2). The psychosomatic therapists received an intensive training focused on PSS and a standardised treatment protocol prior to the study. Psychosomatic therapy according to the standardised treatment protocol consisted of (1) psychosomatic education; (2) bodyoriented exercises; (3) cognitive-behavioural approaches and (4) activating therapy. The therapy was delivered in 6-12 individual sessions of $30-45 \mathrm{~min}$ each. To provide personalised care, therapists were allowed to adjust the intensity, frequency, and sequence of therapy elements. A more detailed description of the psychosomatic therapy (see online supplemental appendix 3) and methods of the CORPUS trial has been published elsewhere. ${ }^{19}$

The current study focuses on the qualitative part of the process evaluation of the CORPUS study. In order to obtain as rich in-depth data as possible, we conducted semistructured (in-depth) face-to-face interviews with patients who received psychosomatic therapy as well as their psychosomatic therapists. In addition to the interviews, we conducted two additional focus groups with psychosomatic therapists. We did not conduct focus groups with patients because this might be a threatening context for patients with PSS, thus preventing patients to speak freely about their experiences with regard to their symptoms and therapy.

Written informed consent was obtained from all participating patients and therapists. Patients and therapists were able to withdraw their consent at any time.

This report was produced using the Standards for Reporting Qualitative Research reporting guidelines. ${ }^{23}$

\section{Patient and public involvement}

Patients or the public were not actively involved in the design, conduct, reporting or dissemination plan of this research.

\section{Participants}

Patients: interviews

With each patient we conducted two semi-structured face to face interviews which took place after completion of psychosomatic therapy (between October 2019 and June 2020) and approximately 1 year later (between March 2020 and November 2020). To select patients for the interviews we used a purposive sampling strategy ${ }^{24}$ to maximise variation sampling to ensure a wide variety of participations in gender, age, region, symptom intensity, number of treatment sessions and therapist. Twenty-five patients were contacted by phone. One patient refused because of lack of time, one patient stopped psychosomatic therapy and did not want to be interviewed, one patient cancelled the first interview appointment for unknown reasons and two patients refused because of personal reasons. So, 20 patients agreed to participate in the first interview. Three patients declined to participate in the final interview because of personal reasons. 
All interviews were conducted by MSHW (psychosomatic therapist and epidemiologist, main researcher) with SD (health scientist, research assistant) or a student (Exercise Therapy or Psychosomatic Physical Therapy) at the research department of the university, at the patients' home or via a Zoom meeting, according to the preference of the patient. All interviewers were female. The first interview had a mean duration of $39 \mathrm{~min}$ (range: $20-59 \mathrm{~min}$ ) and the final interview of $24 \mathrm{~min}$ (range: 16-32 min). During the first interview we used a topic list based on the Medical Research Council guidance ${ }^{25}$ which emphasises the relations between implementation, experiences with the intervention, mechanisms and context of the intervention. Based on the topic list, we developed an interview guide with semistructured, open-ended questions (see online supplemental appendix 4). The interview guide, which was pilot tested, was checked throughout the interview process, and only a few adjustments were made. The second interview started with the following question: Looking back now, what do you think you learned from the therapy? In both interviews we encouraged patients to talk freely about their experiences with and views on the psychosomatic therapy and to expand on any aspect of the therapy that they felt relevant.

The first three participants received a summary of their interview for a member check. ${ }^{26}$ They confirmed that the summary reflected their views, feelings and experiences.

\section{Therapists: interviews}

All therapists who treated one or more patients in the CORPUS study with psychosomatic therapy $(n=25)$ were contacted by phone and asked for participation in a semistructured interview. All therapists agreed to participate. The semi-structured interviews with the therapists took place between November 2019 and June 2020 in the practice of the therapists or via a Zoom meeting. The interviews had a mean duration of $48 \mathrm{~min}$ (range: 33-68 min). We developed a topic list as guidance for the interviews based on (1) audio recordings of therapy sessions made by therapists as part of the CORPUS study, (2) treatment reports of all treated patients made by therapists as part of the CORPUS study and (3) prior research ${ }^{131418}$ Based on the topic list, an interview guide with semi-structured, open-ended questions was formulated (see online supplemental appendix 5). We checked the interview guide after each interview, only some small adjustments were necessary.

\section{Therapist: focus groups}

We decided to conduct two focus groups with psychosomatic therapist in addition to the interviews as this provides added value to study because in a focus group, therapists can respond to each other's input, which is believed to highlight differences as well as similarities in each other's point of view and experiences. ${ }^{27}$

We aimed to select a purposive sample of therapists in order to increase the external validity of the results. ${ }^{24} \mathrm{We}$ aimed at variation in age, gender, years of experience treating PSS patients and professional education (ie, qualified psychosomatic physical or-exercise therapist).

Therapists were invited by email. Six therapists in Amsterdam and seven therapists in Nijmegen agreed to participate. The focus groups took place in January 2020 at the research department of the University Medical Centres of Amsterdam and Nijmegen and lasted $69 \mathrm{~min}$ and $78 \mathrm{~min}$, respectively. Both focus groups were moderated by an independent researcher, retired GP with a specific interest in PSS and experience in moderating focus groups. Furthermore, MSHW and a medical student) were present in the role of observer. The content of the focus groups was based on an interview guide (see online supplemental appendix 6) with semistructured, open-ended questions derived from (1) relevant literature $^{131418}$ and (2) audiorecordings of multiple therapy sessions and individual interviews with participating therapists.

\section{Data analysis}

All interviews and focus groups were audio-recorded and transcribed verbatim. The process of analysis was an iterative process: the researchers (MSHW with SD or a student) started with analysis after the first interviews and focus group to further explore and validate emerging themes in the next interviews. Data were analysed based on thematic analysis according to the six phases described by Braun and Clarke. ${ }^{28}$ In the first phase, we familiarised with the data by reading and rereading the transcripts. In the second phase (generating initial codes), two data coders (MSHW with SD or a student) coded the first three transcripts independently. We coded as many potential topics as possible, using open codes. After initial coding, we discussed any differences in coding in a consensus meeting. Then all interviews were analysed following the same strategy, resulting in an extended open code list. In phases 3 and 4 , we searched for categories and themes. Themes were clustered, reviewed and checked on patterns. In phase 5 , we discussed, defined and named themes, and in an ongoing analysis we refined the specifics of each final theme. In several meetings with the research team, the themes were continuously discussed until agreement was reached on all themes. Finally, the report was produced. Relevant quotes were highlighted and all themes were coded using MAXQDA software. ${ }^{29}$ The analysis of the 20th patient interview yielded no new codes; therefore saturation was reached.

\section{RESULTS}

Thirty-seven patient interview transcripts, 25 therapists interview transcripts and 2 focus groups transcripts were included in the analysis. The characteristics of the participating patients and therapists are summarised in tables 1 and 2 , respectively. 


\begin{tabular}{|c|c|}
\hline Characteristics & $(n=20)$ \\
\hline \multicolumn{2}{|l|}{ Gender } \\
\hline Male & 7 \\
\hline Female & 13 \\
\hline Age in years, mean (range) & $52(24-80)$ \\
\hline \multicolumn{2}{|l|}{ Education } \\
\hline Lower educational level & 6 \\
\hline Intermediate educational level & 6 \\
\hline Higher educational level & 8 \\
\hline Somatic Symptom Score (PHQ-15), mean (range) & $14.8(6-24)$ \\
\hline Symptom intensity, ${ }^{*}$ mean (range) & $6.3(2-10)$ \\
\hline \multicolumn{2}{|l|}{ Main symptoms } \\
\hline Musculoskeletal symptoms & 13 \\
\hline Pain & 16 \\
\hline Shortness of breath & 2 \\
\hline Fatigue & 7 \\
\hline Headache & 5 \\
\hline Dizziness/nausea & 4 \\
\hline Sleep disturbances & 3 \\
\hline No of treatment sessions, mean (range) & $9(3-12)$ \\
\hline
\end{tabular}

PHQ-15 screening: exclusion score $<5$.

*0-10; from no symptoms to most severe symptoms.

PHQ-15, Patient Health Questionnaire-15.

\section{Patients' perspective}

We could identify three main themes regarding the working mechanisms of psychosomatic therapy according to patients: (1) continuous alternation of psychosocial conversations and body-oriented exercises; (2) awareness of body-mind connection and (3) good relationship with therapist.

Continuous alternation of psychosocial conversations and bodyoriented exercises

A majority of the patients described the psychosomatic therapy as an effective combination of a continuously alternation of psychosocial conversation and bodyoriented exercises. According to these patients, the exercises and psychosocial conversations were aimed at (body) awareness, relaxation and at gaining tools to cope with their symptoms.

Psychosomatic therapy is the best of both worlds. There is talking and there's the exercises to really feel what is happening and to relax, and to dare to feel what is actually happening emotionally. [...] For me, that combination of conversation, talking and exercises really is a golden combination that you don't see enough in other therapies. (P2)

Their problems and symptoms were discussed and explored. Most patients told that the therapist explained how symptoms could originate and pointed to the connection between body and mind. Talking and reflecting on their symptoms and problems during and after the treatment sessions seemed to help patients to understand their symptoms. Furthermore, they mentioned that applying the exercises after the session at home was particularly helpful.

And that I was given exercises and could also do the exercises at home. Especially the relaxation exercises, because when your tension was, like, too high, you also remembered, yes, turn the switch, do the exercises. And then you very quickly feel that it works (P4).

Most patients reported that the therapist provided structure, clarity, safety and guidance in the treatment session. In addition, a couple of patients mentioned the calm and steady construction of the treatment sessions.

The therapy was very well structured, from calming down, to spirituality with the meditation exercises and the awareness exercises, then to very slowly building up movement again. (P4)

\section{Awareness of body-mind connection}

Most patients reported that they obtained an awareness of body-mind connection due to the body-oriented exercises. They mentioned that they gained insight and learnt to cope with their symptoms and problems. According to the patients, these experiences of awareness combined

Table 2 Therapists' characteristics

\begin{tabular}{llll}
\hline Characteristics & Interviews ( $\mathbf{n}=\mathbf{2 5})$ & Focus group I (n=6) & Focus group II (n=7) \\
\hline Gender & & & 1 \\
$\quad$ Male & 2 & 1 & 6 \\
$\quad$ Female & 23 & 5 & $51(36-64)$ \\
Age in years, mean (range) & $50(29-65)$ & $50(37-65)$ & 5 \\
Profession & & 2 & 2 \\
$\quad$ Exercise therapist & 12 & 4 & $8.5(2-12)$ \\
\hline Physical therapist & 13 & $8(3-10)$ & \\
Years of work experience with PSS, mean (range) & $9(1-20)$ & & \\
\hline
\end{tabular}

PSS, persistent somatic symptoms. 
with perceived recovery of symptoms led to behavioural change and improved self-regulation.

She tried to provide insight into how you can deal with those symptoms, for example by looking at yourself and asking yourself, what are the triggers? The triggers, the things that directly affect your being, how do you deal with them? And take better care of myself. You first have to inderstand how it works and you have to be willing to accept and come to some kind of behavioural change. (P7)

But a few days after that [session] the insight was there, so to speak. It had sunk in more and then I also appreciated it. (P8)

\section{Good relationship with therapist}

All patients mentioned the importance of a good treatment relationship with the therapist in which therapists provided safety, trust and expertise. They emphasised the necessity of having a connection, in which the therapist is taking time, is listening attentively, without judgement, and is genuinely interested. In addition, most patients experienced that they were taken seriously. They felt understood, respected and treated as an equal partner, they were being heard, seen and validated.

Being able to tell your story, which was very nice by the way, that helped a lot. (P3)

The connection was there, it was there right away actually. And she listened to me and didn't push me. What I could handle, that was our starting point. (P4)

I thought there was good interaction, in which we just tried out many things ... a little more focus on looking for what should be the answer and what should work, only we didn't find that. (P17)

\section{Therapists' perspective}

All therapists described the treatment as a tailored patient-centred process-based approach in which there is a continuous alternation between treatment and exploration of patients' symptoms, perceptions and coping problems during each session.

We could identify four main themes as working mechanisms of the psychosomatic therapy according to therapists: (1) building rapport; (2) continuously searching for common ground; (3) making patients aware of the interaction between body and mind; and (4) continuous alternation between exploration and treatment.

\section{Building rapport}

All therapists mentioned that establishing contact and building rapport with the patient is their first priority. Therapists experienced building rapport as a prerequisite for a good therapeutic alliance and as an working mechanism of the psychosomatic therapy. They explained that they use several techniques to build rapport with their patient. This starts immediately during the intake and continues throughout the treatment. They attempt to build rapport by genuinely listening attentively to their story, without judgement while being attentive to possible cues about underlying issues embedded within the story. Therapists mentioned that providing structure, safety and guidance during treatment is important for a successful psychosomatic therapy. There is a continuous exchange between following and leading of the patient. The therapists always use communication skills as summarising, positive labelling, challenging and confronting the patient.

Making the patient feel heard, that's important, that's where it starts, making a connection. Acknowledging the resistance, which I do, and I explain that I can understand where that's coming from considering their history; everything the person has already been through that hasn't helped. Show that there is also understanding for their resistance. (T3)

\section{Continuously searching for common ground}

Most therapists indicated it is essential that the patient and therapist agree about the explanation of the symptoms and that they try to find common ground on the psychosomatic approach which is acceptable to both patient and therapist while making sure there are no unrealistic expectations. In addition, they mentioned that the patient's willingness to change is conditional for finding common ground.

That people are willing to view their symptoms from a different perspective than what they're used to. [...], so that they're willing to explore what else it could be. That they're curious about it and their own role in it, like how does it actually work and what can I do with that information. To me, that's an ideal patient. (T22)

Making patients aware of the interaction between body and mind All therapists indicated that they apply all four therapy elements: psychosomatic education, body-oriented exercises, cognitive behavioural approaches and at home exercises in order to make patients aware of the bodymind interaction. The choice and to what extent a certain therapy element is involved in the patient's treatment depends on the patient's symptoms, characteristics, capabilities, request for help and risk factors identified with the patient as well as the personal preference and experience of the therapist.

According to the therapists, there is no standard protocol for psychosomatic therapy for patients with PSS nor is it possible to design one.

Doing exercises, making your client aware of tension in his body, making him feel the difference and explaining a lot. Finding out together with your client, what is conducive to recovery, what would be good for her to do. (T6)

Therapists explained that physical and emotional awareness is one of the major themes during therapy. 
Besides using psychosocial conversation with psychoeducation on the interaction between body and mind and the influence of this interaction on their physical symptoms, CBT techniques are used, to gain insight into the patient's cognitions and to clarify the relationship between patients' thinking, feeling and doing. A majority of the therapists mentioned to prefer body-oriented therapy with body-awareness exercises to help their patients to literally feel what is happening within their body. They try to learn the patients to experience how physical symptoms can influence their mind, emotions and behaviour and vice versa in order to improve selfmanagement of their symptoms.

Many of the people we treat are not conscious of their body, so there is no connection between what they experience in relation to the body. To use the body, which I think we all do, to get to this experience is the biggest challenge. It can be done by trying to get people to exercise and by making people feel. (T13)

All therapists emphasised that they believe that their approach of using the body as entry point in treating PSS helping patients to understand both the physical and emotional aspects of their symptoms, is one of the strongest qualities of psychosomatic therapy.

\section{Continuous alternation between exploration and treatment}

Most therapists mentioned that they continuously alter between exploration and treatment. They mentioned the importance of starting each treatment period with establishing the patient's request for help and of treatment goals. During the treatment process, the therapist monitors the previously established treatment goals together with the patient.

Start with formulating the request for help, the goals and then return to them again and again.

At some point I think they'll have enough tools to go on under their own steam. (T7)

In addition, therapists described that each treatment session starts with questions focused on the patient's symptoms and their course in the past period. If necessary, more in-depth questions are asked for further exploration such as somatic, cognitive, emotional, behavioural and social aspects of the patients' symptoms. In addition, therapists mentioned that they discuss and explore patients' predisposing factors (individual characteristics of a patient), precipitating (triggering) factors and perpetuating (maintaining) factors as possible explanation of the symptoms.

I was still looking for the perpetuating factors with him in order to explain it biopsychosocially, psychosomatically (...) and I could explain it better in terms of predisposing, perpetuating and precipitating factors to steer the patient towards the psychosomatic path. (T21)

\section{DISCUSSION}

Patients and therapists stress the value of the combination of psychosocial conversations and body-oriented exercises and they highlight the importance of awareness of a body-mind interaction to understand the physical as well the emotional aspects of patients' symptoms. Achieving a positive therapeutic alliance and finding common ground with regard to the treatment are prerequisites for the success of the treatment. Therapists apply various tools to build rapport and, as a result, patients experience being taken seriously, being heard, seen and validated. Therapists emphasise that they apply all four therapy elements in varying combinations based on patient preferences, patient knowledge, therapist preferences and the continuous exploration of the problem at hand.

As far as we know, no prior qualitative study focused on the opinions and views from therapists' and patients' perspective on the elements contributing to the effects of psychosomatic therapy in patients with PSS. Triangulation of sources were used to collect and analyse the data, following an iterative process and modification of procedures in response to evolving study findings.

Some limitations should be taken into account. First, during this study the COVID-19 pandemic started. Due to this crisis in some cases the continuity of the therapy was interrupted which might have affected patients' experience with psychosomatic therapy. In addition, we conducted a number of interviews via Zoom meetings. No participant indicated that they did not like the online interview, but the interview was sometimes challenging due to unstable internet connection and poor sound quality. Second, usually patients visit their GP with a specific request for help regarding their symptoms. The GP provides explanations about the patient's symptoms and about how psychosomatic therapy might be of help for them when he/she refers patients to psychosomatic therapy. In the CORPUS study, patients were identified by a search strategy from the electronic medical records. Patients received a letter from their GP, with brief information about the CORPUS study and therapy, but the usual preparation of psychosomatic therapy by their GP lacked. Therefore, a majority of the participants indicated that they started the therapy without expectations and did not always seem to expect a psychosomatic approach. This may have influenced their experiences with the psychosomatic therapy. Third, it is remarkable that neither patients nor therapists mentioned potential downsides, harms or adverse effects of psychosomatic therapy. It is uncertain whether this was because there simply were none or because people gave socially desirable answers. Finally, our exclusion criterion of 'insufficient understanding of the Dutch language' led to less variation in terms of ethnicity of the participants of our study.

Our findings correspond to previous research stating that treatment in patients with PSS should be biopsychosocial and multimodal, as well as activating, patient-involving and operating on the basis of a biopsychosocial model of integrating somatic as well as psychosocial determinants of distress and 
therapeutic factors. ${ }^{30} 31$ Our study shows that according to patients and therapists, the combination of body-oriented exercises and psychosocial conversations is very valuable in which particularly gaining awareness of the body-mind interaction $^{32}{ }^{33}$ is important. A recent study on treatment modalities in a multidisciplinary and blended care intervention in primary care identified some similar themes such as relaxation, body awareness exercises and graded activity which also emerge as important elements of the treatment in our study. ${ }^{34}$ In a recent synthesis of qualitative evidence of behavioural modification interventions for medically unexplained symptoms in primary care Leaviss et alsuggested that the quality of relationship between the service user and their GP is vital, and this 'therapeutic alliance' is the determining factor for how successfully these interventions can be. ${ }^{11} \mathrm{~A}$ key factor in whether or not a patient will engage with, and ultimately benefit from, a treatment is whether or not they believe that their concerns are being taken seriously and that their feelings are being respected. ${ }^{11}$ This is in line with our findings that a good therapeutic alliance is a prerequisite for the success of psychosomatic therapy.

A recent study on health interventions for chronic pain suggested that interventions should focus on validating pain through meaningful and rational explanations and validating patients by listening to and valuing their stories. ${ }^{35} \mathrm{In}$ our study, therapists as well as patients mentioned the importance of these issues. However, the most important elements in providing symptom explanation according to therapists in our study is the body awareness exercise through which patients experience the body-mind interaction themselves.

According to research with a facilitated support group for patients with PSS, ${ }^{36}$ patients mentioned that they feel a gap in currently available clinical care offered by healthcare professionals and feel validated through sharing similar experiences with peers. In our study no participant mentioned the lack of supporting of peers.

As finding common ground regarding patient's symptoms and a psychosomatic approach is essential, it will be helpful that the GP prepare and inform the patient about the psychosomatic approach. Conveying realistic expectations regarding the treatment may result in patients to be receptive to such an approach. Good interdisciplinary cooperation and communication between the therapist and the GP seems to be important, which is confirmed by previous research, ${ }^{37}$ but further research into the influence of interdisciplinary cooperation and communication on the results in the treatment of patients with PSS is needed.

Our findings regarding psychosomatic therapy for patients with PSS may also be applicable to regular exercise therapy and physical therapy for patients with other symptoms, but further research will be necessary.

\section{Conclusion}

According to patients as well as therapists, the continuous alternation of psychosocial conversations and bodyoriented exercises to provide awareness of the interaction between body and mind are the perceived working mechanism of psychosomatic therapy. Therapeutic alliance and finding common ground between patient and therapist are prerequisites for the success of psychosomatic therapy.

Acknowledgements We want to thank all the patients and therapists who took part in this study for sharing their experiences and opinions. Special acknowledgement to Peter Lucassen for moderating the focus groups and to the bachelor and master students, who participated in the data collection as part of their Bachelor of Exercise therapy, Master of Psychosomatic Physiotherapy or Master of Medicine.

Contributors MSHW, TCOH, JCvdW and HEvdH developed the study protocol. WJJA and BV provided feedback on the study protocol. MSHW developed the topic guide, MSHW and SD collected and analysed the data and interpreted the results. TCOH provided feedback on the topic guide, data collection, analysis and interpretation. MSHW drafted the manuscript. All authors critically and substantially revised the manuscript and approved the current version to be submitted for publication. MSHW is acting as guarantor.

Funding This study was funded by the Stoffels-Hornstra Foundation (Dutch Foundation) and ZonMw (grant number 843001802). MSHW obtained a personal grant (023008010) from the Netherlands Organisation for Scientific Research (NW0).

Disclaimer The funding sources had no role in the design, collection, analysis or interpretation of data, nor in the writing of the manuscript.

Competing interests None declared.

Patient consent for publication Not applicable.

Ethics approval This study was approved by The Medical Ethics Committee of VU University Medical center (METc VUmc) approved the research protocol; ID 2018.011.

Provenance and peer review Not commissioned; externally peer reviewed.

Data availability statement No data are available.

Supplemental material This content has been supplied by the author(s). It has not been vetted by BMJ Publishing Group Limited (BMJ) and may not have been peer-reviewed. Any opinions or recommendations discussed are solely those of the author(s) and are not endorsed by BMJ. BMJ disclaims all liability and responsibility arising from any reliance placed on the content. Where the content includes any translated material, BMJ does not warrant the accuracy and reliability of the translations (including but not limited to local regulations, clinical guidelines, terminology, drug names and drug dosages), and is not responsible for any error and/or omissions arising from translation and adaptation or otherwise.

Open access This is an open access article distributed in accordance with the Creative Commons Attribution Non Commercial (CC BY-NC 4.0) license, which permits others to distribute, remix, adapt, build upon this work non-commercially, and license their derivative works on different terms, provided the original work is properly cited, appropriate credit is given, any changes made indicated, and the use is non-commercial. See: http://creativecommons.org/licenses/by-nc/4.0/.

ORCID iDs

Margreet S H Wortman http://orcid.org/0000-0002-4799-3041

Henriëtte E van der Horst http://orcid.org/0000-0003-4060-4354

\section{REFERENCES}

1 Verhaak PFM, Meijer SA, Visser AP, et al. Persistent presentation of medically unexplained symptoms in general practice. Fam Pract 2006;23:414-20.

2 den Boeft M, Twisk JWR, Hoekstra T, et al. Medically unexplained physical symptoms and work functioning over 2 years: their association and the influence of depressive and anxiety disorders and job characteristics. BMC Fam Pract 2016;17:46.

3 Joustra ML, Janssens KAM, Bültmann U, et al. Functional limitations in functional somatic syndromes and well-defined medical diseases. results from the general population cohort lifelines. J Psychosom Res 2015;79:94-9.

4 Dirkzwager AJE, Verhaak PFM. Patients with persistent medically unexplained symptoms in general practice: characteristics and quality of care. BMC Fam Pract 2007;8:33.

5 Sattel H, Lahmann C, Gündel H, et al. Brief psychodynamic interpersonal psychotherapy for patients with multisomatoform disorder: randomised controlled trial. Br J Psychiatry 2012;200:60-7. 
6 Menon V, Rajan TM, Kuppili PP, et al. Cognitive behavior therapy for medically unexplained symptoms: a systematic review and meta-analysis of published controlled trials. Indian J Psychol Med 2017;39:399-406.

7 van Dessel N, den Boeft M, van der Wouden JC, et al. Nonpharmacological interventions for somatoform disorders and medically unexplained physical symptoms (MUPS) in adults. Cochrane Database Syst Rev 2014;11:CD011142.

8 Kleinstauber M, Witthoft M, Steffanowski A. Pharmacological interventions for somatoform disorders in adults. Cochrane Database Syst Rev 2014;11:CD010628.

9 Abbass A, Kisely S, Kroenke K. Short-Term psychodynamic psychotherapy for somatic disorders. systematic review and metaanalysis of clinical trials. Psychother Psychosom 2009;78:265-74.

10 Martens U, Enck P, Matheis A, et al. Motivation for psychotherapy in patients with functional gastrointestinal disorders. Psychosomatics 2010;51:225-9.

11 Leaviss J, Davis S, Ren S, et al. Behavioural modification interventions for medically unexplained symptoms in primary care: systematic reviews and economic evaluation. Health Technol Assess 2020;24:1-490.

12 Katsamanis M, Lehrer PM, Escobar Jl, et al. Psychophysiologic treatment for patients with medically unexplained symptoms: a randomized controlled trial. Psychosomatics 2011;52:218-29.

13 Olde Hartman TC, Rosendal M, Aamland A, et al. What do guidelines and systematic reviews tell us about the management of medically unexplained symptoms in primary care? BJGP Open 2017;1:bjgpopen17X101061.

14 Heijmans M, Olde Hartman TC, van Weel-Baumgarten E, et al. Experts' opinions on the management of medically unexplained symptoms in primary care. A qualitative analysis of narrative reviews and scientific editorials. Fam Pract 2011;28:444-55.

15 Mulders N, Boersma R, ljntema R. Vocational competence profile psychosomatic physical therapist [In Dutch: Beroepscompetentieprofiel Psychosomatisch Fysiotherapeut]. Amersfoort: NFP, 2009

16 VvOCM. Vocational competence profile psychosomatic exercise therapist [in Dutch: Beroepsprofiel Psychosomatisch Oefentherapeut]. VvOCM: Utrecht, 2020.

17 Olde Hartman TC, Blankenstein AH, Molenaar AO. Dutch college of GP guideline on medically unexplained symptoms (MUS) [In Dutch NHG-standaard Somatisch Onvoldoende verklaarde Lichamelijke Klachten (SOLK)]. Huisarts en Wetenschap 2013;5:8

18 Wortman MSH, Lucassen PLBJ, van Ravesteijn HJ, et al. Brief multimodal psychosomatic therapy in patients with medically unexplained symptoms: feasibility and treatment effects. Fam Pract 2016;33:346-53.

19 Wortman MSH, van der Wouden JC, Grutters JPC, et al. Psychosomatic therapy for patients frequently attending primary care with medically unexplained symptoms, the CORPUS trial: study protocol for a randomised controlled trial. Trials 2019;20:697.

20 Robbins JM, Kirmayer LJ, Hemami S. Latent variable models of functional somatic distress. J Nerv Ment Dis 1997;185:606-15.
21 Kroenke K, Spitzer RL, Williams JBW. The PHQ-15: validity of a new measure for evaluating the severity of somatic symptoms. Psychosom Med 2002;64:258-66

22 van Ravesteijn $\mathrm{H}$, Wittkampf $\mathrm{K}$, Lucassen $\mathrm{P}$, et al. Detecting somatoform disorders in primary care with the PHQ-15. Ann Fam Med 2009;7:232-8.

23 O'Brien BC, Harris IB, Beckman TJ, et al. Standards for reporting qualitative research: a synthesis of recommendations. Acad Med 2014;89:1245-51.

24 Boeije H. Analysing in qualitative research [In Dutch:Analyseren in kwalitatief onderzoek: denken en doen]. Den Haag: Boom/Lemma, 2014.

25 Moore GF, Audrey S, Barker M, et al. Process evaluation of complex interventions: Medical Research Council guidance. BMJ 2015;350:h1258.

26 Cutcliffe JR, McKenna HP. When do we know that we know? Considering the truth of research findings and the craft of qualitative research. Int J Nurs Stud 2002;39:611-8.

27 Doody O, Slevin E, Taggart L. Focus group interviews in nursing research: part 1. Br J Nurs 2013;22:16-19.

28 Braun V, Clarke V. Using thematic analysis in psychology. Qual Res Psychol 2006;3:77-101.

29 MAXQDA 2020 [computer software]. Berlin: VERBI Software, 2020. Available: https://www.maxqda.com

30 Henningsen P, Zipfel S, Herzog W. Management of functional somatic syndromes. Lancet 2007;369:946-55

31 Henningsen P, Zipfel S, Sattel H, et al. Management of functional somatic syndromes and bodily distress. Psychother Psychosom 2018;87:12-31.

32 De Berardis D, Fornaro M, Orsolini L. Editorial: 'No words for feelings, yet!' exploring alexithymia, disorder of affect regulation, and the 'mind-body' connection. Front Psychiatry 2020;11:593462.

33 Motofei I, Rowland D. The mind-body problem; three equations and one solution represented by immaterial-material data. JMMS 2018;5:59-69.

34 van Westrienen PE, Pisters MF, Gerrits M, et al. Identifying treatment modalities for a multidisciplinary and blended care intervention for patients with moderate medically unexplained physical symptoms: qualitative study among professionals. JMIR Ment Health 2019;6:e12203.

35 Toye F, Belton J, Hannink E, et al. A healing journey with chronic pain: a Meta-Ethnography synthesizing 195 qualitative studies. Pain Med 2021;22:1333-44.

36 Marcinow M, Sandercock J, D'Silva C, et al. Making sense of symptoms, clinicians and systems: a qualitative evaluation of a facilitated support group for patients with medically unexplained symptoms. BMC Fam Pract 2021;22:142.

37 Balabanovic J, Hayton P. Engaging patients with "medically unexplained symptoms" in psychological therapy: An integrative and transdiagnostic approach. Psychol Psychother 2020;93:347-66 